\title{
A Placement and Routing Method for Layout Generation of CMOS Operational Amplifiers Using Multi-Objective Evolutionary Algorithm Based on Decomposition
}

Mehran Nohtanipour ${ }^{1}$, Mohammad Hossein Maghami ${ }^{*}, 2$, Mehdi Radmehr ${ }^{1}$

${ }^{1}$ Department of Electrical Engineering, Sari Branch, Islamic Azad University, Sari, Iran ${ }^{2}$ Faculty of Electrical Engineering, Shahid Rajaee Teacher Training University, Tehran, Iran

Abstract: This paper presents a new placement and routing method for layout generation of CMOS operational amplifiers (op-amps). Both circuit sizing and layout generation stages are performed automatically. In the proposed method, layout effects are considered during the layout generation. Layout parasitics and geometry information are extracted from a new automated layout generator. In this method, the multi-objective evolutionary algorithm based on decomposition (MOEA/D) is used as an optimization algorithm. In order to verify the performance of the proposed method, the design of three-stage operational amplifier (op-amp) and two-stage class-AB operational trans-conductance amplifier (OTA) in a $0.18 \mu \mathrm{m}$ process CMOS technology with $1.8 \mathrm{~V}$ supply voltage are presented. The simulation results indicate the efficiency of the proposed analog layout generation method.

Keywords: Analog layout generation; Circuit sizing; Automated placement and routing; MOEA/D; Three-stage operational amplifier

\section{Metoda umeščanja in usmerjanja za generiranje postavitve operacijskih ojačevalnikov CMOS z uporabo večciljnega evolucïskega algoritma na osnovi dekompozicije}

\begin{abstract}
Izvleček: Članek predstavlja novo metodo umeščanja in usmerjanja za izdelavo postavitve operacijskih ojačevalnikov CMOS (opamperov). Fazi določanja velikosti vezja in generiranja postavitve se izvajata samodejno. Pri predlagani metodi se med generiranjem postavitve upoštevajo učinki postavitve. Parazitske lastnosti postavitve in informacije o geometriji se pridobijo iz novega samodejnega generatorja postavitve. $\vee$ tej metodi se kot optimizacijski algoritem uporablja večobjektni evolucijski algoritem, ki temelji na dekompoziciji (MOEA/D). Da bi preverili učinkovitost predlagane metode, sta predstavljeni zasnova tristopenjskega operacijskega ojačevalnika (op-amp) in dvostopenjskega operacijskega ojačevalnika razreda AB (OTA) v tehnologiji CMOS z 0,18 $\mu m$ procesom in napajalno napetostjo 1,8 V. Rezultati simulacije kažejo učinkovitost predlagane metode generiranja analogne postavitve.
\end{abstract}

Ključne besede: Generiranje analogne postavitve; Določanje velikosti vezja; Avtomatizirano umeščanje in usmerjanje; MOEA/D; Tristopenjski operacijski ojačevalnik

*Corresponding Author's e-mail:mhmaghami@sru.ac.ir

\section{Introduction}

Analog circuit design includes three main steps as follows [1], [2], [3], [4]: topology synthesis / selection, circuit sizing and layout design. In the first step, a proper circuit topology is selected by a designer. The second step is sizing devices with the aim of finding proper width and length of the transistors, passive components values, 
bias voltages and currents. Finally, the layout should be generated from the previous step. In the analog integrated circuit (IC) design, it is often required to optimize different specifications simultaneously. Designing the circuit can be performed manually which is a difficult task and takes lots of time. There are large number of circuit sizing methods in the literature [5], [6], [7], [8], [9]. In the knowledge-based approach, sizes of circuit devices are determined based on some pre-defined design flows and databases. However, this method is time-consuming and there is no guarantee to convergence to the global optimum solution. Optimization-based methods can be divided into three categories as follows: "equation-based", "simulation-based" and "equation and simulation-based" methods [10], [11]. In the equation-based method, mathematical solvers are used to solve the circuit equations and satisfy the target specifications. In the simulation-based method, a circuit simulator is used to evaluate the target specifications for a set of design variables. Finally, to have a compromise between speed and accuracy of the two mentioned methods, equation and simulation-based method was utilized in some designs [12].

Automatic analog layout generation has been considered by analog circuits designers [13]. In the literature, several Computer-Aided Design (CAD) tool [14], [15]. shave been introduced for analog circuits layouts. However, circuit sizing and layout generation steps are not regarded simultaneously. Since circuit performance can be influenced by layout parasitics, the circuit specifications may not be satisfied after post-layout simulation. The behavior of analog integrated circuits is sensitive to layout-induced parasitics. To achieve desired specifications, time-consuming and non-systematic iterations between these circuit sizing and layout synthesis are required [16]. If these issues are not taken into account, circuit overdesign may occur which results in wasting power and area. On the other hand, circuit underestimation may lead to a worsening of post-layout performance. It is useful to perform circuit sizing and layout generation steps automatically.

Some approaches have been proposed in the analog circuit domain to assist the designers. But some procedures should be performed manually. Optimizationbased sizing approaches are employed for analog IC design automation using the circuit simulators as evaluation engines [17], [18]. The quality of a design is evaluated by the degree to which constraints are satisfied and also desired specifications are achieved. In order to achieve the better sizing results, the layout effects should be considered. The layout-aware sizing method consists of parasitic-aware sizing and geometry-aware sizing [19], [20]. In parasitic-aware sizing method, parasitics of the layout are extracted continuously and then they are utilized in the sizing process. In the geometryaware sizing method, the value of geometrical parameters such as width, length, and the number of fingers of MOS transistors are chosen such that the layout area and shape to be optimized. Several analog layout-aware sizing methods have been introduced in the literature.

Parametric generators are used that code the whole layout of the circuit to increase execution speed. However, their definition is time-consuming and the solutions for devices' sizes may differ from the ones intended in the definition step [21], [22], [23]. In Ref [24], a predefined floorplan template supported by a slicing tree is used. To obtain the desired geometric features, the number of fingers of MOS transistors should be adjusted. A similar idea is suggested by minimizing the placement area using convex optimization [25]. However, placement solutions are not compact. In Ref [26], parasitics estimation are performed without actual layout generation. However, these methods suffer from the fixed layout template used for layout generation or parasitic model construction. A method to model the parasitics associated with inductors during RF circuit synthesis is suggested [27]. The post-layout results may not be acceptable since other parasitics are not considered. A method is proposed to estimate parasitics from earlier placement information and includes this during the circuit resizing stage. Since the parasitic information is related to a single design, it does not take the parasitic variation and it may not be sufficient to use in resizing stage [28].

Layout with templates and commercial extractors is used in Ref [29], In this method, symbolic analysis is employed to combine the parasitics with the performance models. But, geometrical information is not taken into account. A parasitic-aware sizing method is suggested in Ref [30], This method is only applicable to a pre-defined floorplan. A placement and routing method for analog layout generation based on a modified cuckoo optimization algorithm (MCOA) is introduced in Ref [31] In this method, layout parasitics are considered to avoid performance deterioration. But, the placement and routing stages have some shortcomings as it will be discussed in this paper. In Ref [32], an automatic method to generate chip floorplans is proposed. The obtained results are superior or comparable to the solutions produced by designers in terms of power consumption, performance and chip area. In order to achieve this aim, chip floorplanning is considered as a reinforcement learning problem. An edge-based graph convolutional neural network architecture is introduced which has the characteristics of learning rich and transferable representations of the chip.

This paper presents a new technique for placement and routing in analog layout generation using the MOEA/D. 
Circuit performance and layout effects are considered in the proposed method. In addition, geometrical information is regarded in the proposed method. The rest of the paper is organized as follows: Multi-objective evolutionary algorithm based on decomposition is introduced in Section 2. The proposed automatic analog layout generation method is described in Section 3. Simulation results are provided in Section 4. Finally, the conclusion is given in Section 5 .

\section{Multi-objective evolutionary algorithm based on decomposition (MOEA/D)}

At present, engineering designs are not usually a simple optimization problem. Multiple objectives are usually desired especially when conflicting operations exist between the optimization searching for different design specifications. During the past decades, evolutionary multi-objective optimization (EMO) has been used by many researchers in the area of intelligent computing [33], [ 34]. EMO algorithms have advantages in exploring a set of Pareto-optimal solutions compared to traditional methods. Many EMO algorithms utilize Pareto dominance for fitness assignment. In these algorithms, the fitness value of each individual is achieved by comparing it with other individuals in terms of Pareto dominance. therefore, all non-dominated solutions in the population should have the best fitness value.

In this paper, multi-objective optimization problems can be considered as follows:

$$
\begin{aligned}
& \operatorname{Minimize}\left(f_{1}(x), f_{2}(x), \ldots, f_{M}(x)\right) \\
& \text { Subject to } g(x) \geq 0, X_{L}<x<X_{H}
\end{aligned}
$$

where $f_{i}(x), i=1 . . M$ is the objective function, $M$ is the number of objectives, $x$ includes design variables, and $X_{L}$ and $X_{H}$ are their lower and upper bounds, respectively. The vector $g(x) \geq 0$ represents the design constraints.

A multi-objective evolutionary algorithm based on decomposition (MOEA/D) has been proposed for multiobjective optimization problem (MOP) [35]. In MOEA/D, a MOP is decomposed into several scalar subproblems in which the optimization is performed simultaneously. Using the information on solutions of neighborhood subproblems, this method has less computational cost, which has been proved by several numerical tests.

The motivation of MOEA/D is to decompose a multiobjective optimization problem into several scalar optimization sub-problems using a scalar function. In this method, optimization is performed simultaneously by the evolutionary algorithm. In MOEA/D, each non dominated solution of the multi-objective optimization problem is related to an optimal solution of the single objective optimization problem and it is computed using a specific weight vector. MOEA/D uses a set of weight vectors to set up different search directions, and different weight vectors will direct to search the different regions of the objective space. The Tchebycheff method can be used to decompose a multi-objective optimization problem into $\mathrm{N}$ sub-problems. In this method, the objective function of the $j$ th $(j=1,2, \ldots$, N) sub-problem is as follows:

$g^{t e}\left(x \mid \lambda^{j}, z^{*}\right)=\max _{1 \leq i \leq m}\left\{\lambda_{i}^{j}\left|f_{i}(x)-z_{i}^{*}\right|\right\}$

where $\lambda^{j}=\left(\lambda_{1}{ }^{j}, \ldots, \lambda_{m}{ }^{j}\right)^{T}$ demonstrates a weight vector, $z^{*}=\left(z_{1}{ }^{*}, \ldots, z_{m}{ }^{*}\right)^{T}$ represents the vector of reference points. For each Pareto optimal point $x^{*}$ there exists a weight vector so that $x^{*}$ is the optimal solution of (2). The methods to determine the weight vector can be found in the related references such as Ref [36]. It should be mentioned that each optimal solution of Eq. (2) is a Pareto optimal solution of the problem of Eq. (1).

\section{Proposed Placement and Routing Method}

In this Section, the proposed placement and routing stages based on the MOEA/D are described.

\subsection{Placement}

In the placement stage, the area of floorplan is optimized by simultaneous consideration of symmetry and proximity constraints. It is necessary to place the devices by considering symmetry and proximity constraints to alleviate the parasitic coupling effects and also to improve circuit performance. Therefore, they are briefly reviewed here [37]. A set of $m$ symmetry groups can be shown by $S=\left\{S_{1}, S_{2}, \ldots, S_{m}\right\}$. The coordinates of the symmetry axes are indicated by $\left(\hat{x}_{i}, \hat{y}_{i}\right)$. A member of the groups is described as follows:

$$
\begin{gathered}
S_{i}=\left\{\left(\mathrm{M}_{1}, \mathrm{M}_{1}^{\prime}\right),\left(\mathrm{M}_{2}, \mathrm{M}_{2}^{\prime}\right), \ldots,\left(\mathrm{M}_{u}, \mathrm{M}_{u}^{\prime}\right)\right. \\
\left., \mathrm{M}_{1}^{s}, \mathrm{M}_{2}^{s}, \ldots, \mathrm{M}_{v}^{s}\right\}
\end{gathered}
$$

This group includes $u$ symmetry pairs $\left(\mathrm{M}_{j}, \mathrm{M}_{j}^{\prime}\right)$ with coordinates centers $\left(\mathrm{x}_{j}, y_{j}\right)$ and $\left(\mathrm{x}_{j}^{\prime}, y_{j}^{\prime}\right)$ and $v$ self-symmetric modules $\mathrm{M}_{k}^{s}$ with coordinate center $\left(\mathrm{x}_{s}^{k}, y_{s}^{k}\right)$. The conditions for symmetry group with vertical symmetric axis are indicated by (4) - (6), and 
equations set (7) - (9) are used for those with horizontal axis.

$$
\begin{aligned}
& \mathrm{x}_{j}+x_{j}^{\prime}=2 \times \hat{x}_{i}, \forall i=1,2, \ldots, \mathrm{u} . \\
& y_{j}=y_{j}^{\prime}, \forall i=1,2, \ldots, \mathrm{u} . \\
& \mathrm{x}_{s}^{k}=\hat{x}_{i}, \forall k=1,2, \ldots, \mathrm{v} . \\
& \mathrm{x}_{j}=x_{j}^{\prime}, \forall i=1,2, \ldots, \mathrm{u} . \\
& y_{j}+y_{j}^{\prime}=2 \times \hat{y}_{i}, \forall i=1,2, \ldots, \mathrm{u} . \\
& y_{s}^{k}=\hat{y}_{i}, \forall k=1,2, \ldots, \mathrm{v} .
\end{aligned}
$$

The proximity condition is described as follows:

$$
\sigma^{2}(\Delta P)=\frac{A_{P}^{2}}{W L}+S_{P}^{2} D_{x}^{2}
$$

where $\Delta P$ is the difference of an electrical parameter $P$, $A_{0}$ is the area proportionality constant for $P, W$ and $L$ are the respective width and length of the device, and $S_{p}$ is the variation of $P$ under the device spacing $D_{x}$. As can be seen from the above equation, it is necessary to place the symmetry group together as close as possible.

For two groups of modules such as A and B, a common centroid constraint is defined as below:

$$
\begin{aligned}
& \sum_{a \in A}\left(x_{a}+\frac{\omega_{a}}{2}\right)=\sum_{b \in B}\left(x_{b}+\frac{\omega_{b}}{2}\right) \\
& \sum_{a \in A}\left(y_{a}+\frac{h_{a}}{2}\right)=\sum_{b \in B}\left(y_{b}+\frac{h_{b}}{2}\right)
\end{aligned}
$$

In the above equations, $(x, y)$ and $(\omega h)$ show the lower left coordinates and the width and height of the modules, respectively.

In addition, design rules should be satisfied at this stage. A method for handling constraints would be to consider them as new objective functions. If $\mathrm{g}(\mathrm{x}) \geq 0$ must hold, for instance, we can transform this to a new objective function $f_{\text {new }}(x)=\min \{-g(x), 0\}$ subject to minimization.

The devices to be placed on the floorplan are represented by $k$ modules $M_{1}, \ldots, M_{k}$. The objective functions are defined for the placement stage as follows:

$$
\begin{aligned}
& f_{P}(\mathrm{x})=\left(f_{o P 1}(x),-f_{o P 2}(x)\right) \\
& f_{o P 1}(x)=\text { Width }_{\text {floorplan }} \times \text { Height }_{\text {floorplan }}
\end{aligned}
$$

$f_{o P 2}(x)=\frac{\text { Total Blocks A rea }}{\text { Width }_{\text {floorplan }} \times \text { Height }_{\text {floorplan }}}$

In the above equation, $x=\left\{\left(x_{1}, y_{1}\right), \ldots,\left(x_{k}, y_{k}\right)\right\}$ indicates the coordinates of the left-bottom corners of the modules, $f_{o P 1}(x)$ is the area of the floorplan. Width floor- $_{\text {. }}$ and Height $t_{\text {floorplan }}$ represent the floorplan width and height, respectively. The term area utilization is defined by $f_{o P 2}(x)$ and it is no greater than 1 . It is worth to mention that the $f_{p}(x)$ is formulated to deal with a minimization optimization problem. In the placement stage, optimization is performed using the MOEA/D.

\subsection{Routing}

The routing stage aims to electrically connect the terminals of the layout devices such as transistors, capacitors, differential pairs. In this proposed method, each wire is divided into d segments. Each segment is represented by segment direction, segment layer. Segment direction can be defined as up, down, left, and right. Segment is located in the layers from 1 to $n$, where $n$ is the number of the layers.

Positions of the segments are adjusted automatically so that the wire length between terminal pairs to be minimized. The objective functions for the routing stage are proposed as follows:

$f_{R}=\left(f_{o R 1}(x), f_{o R 2}(x), f_{o R 3}(x)\right)$

$f_{o R 1}(x)=\sum_{i=1}^{d}$ Length $_{\text {Segment }_{i}}$

$f_{\text {oR } 2}(x)=$ Number of Vias

$f_{o R 3}(x)=$ Number of bends

Where $f_{\text {oRI }}(x)$ is the sum of the segment lengths from the starting terminal $\left(T_{S}\right)$ to the target terminal $\left(T_{E}\right)$ (See Figure1). $\left(x_{T_{S}}, y_{T_{S}}, z_{T_{S}}\right)$ and $\left(x_{T_{E}}, y_{T_{E}}, z_{T_{E}}\right)$ are the coordinates of the starting terminal and the target terminal, respectively. $f_{O R 2}(x)$ is the number of vias in the wire. $f_{O R 3}(x)$ is the number of bends in the wire and it is defined to avoid unnecessary change of direction between adjacent segments. It is worth to mention that it is not considered in the Ref [31]. Current-density to determine the segment width and design rules are the constraints in the routing stage. An example of wiring progress using the proposed method is demonstrated in Figure 2. In this Figure, different segment colors indicate transition between layers.

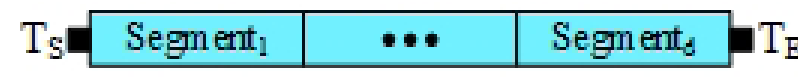

Figure 1: Wire segmentation in the proposed method. 
(a)

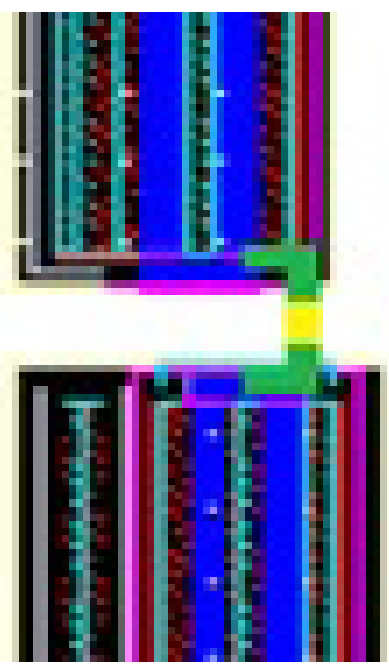

(b)

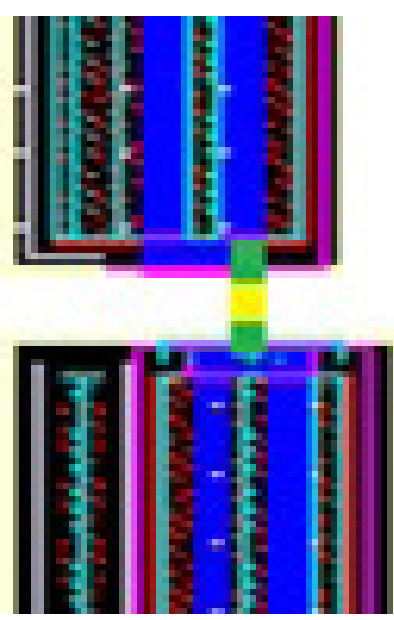

(c)

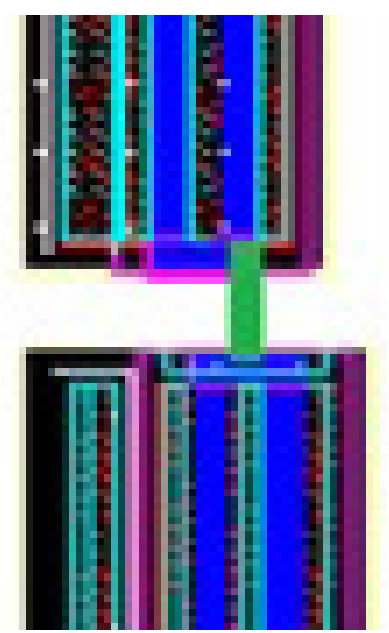

Figure 2: Example of routing stage progress using the proposed method: (a) Wiring between two terminals after 1 generation, (b) Wiring between two terminals after 10 generations, (c) Wiring between two terminals after 100 generations.

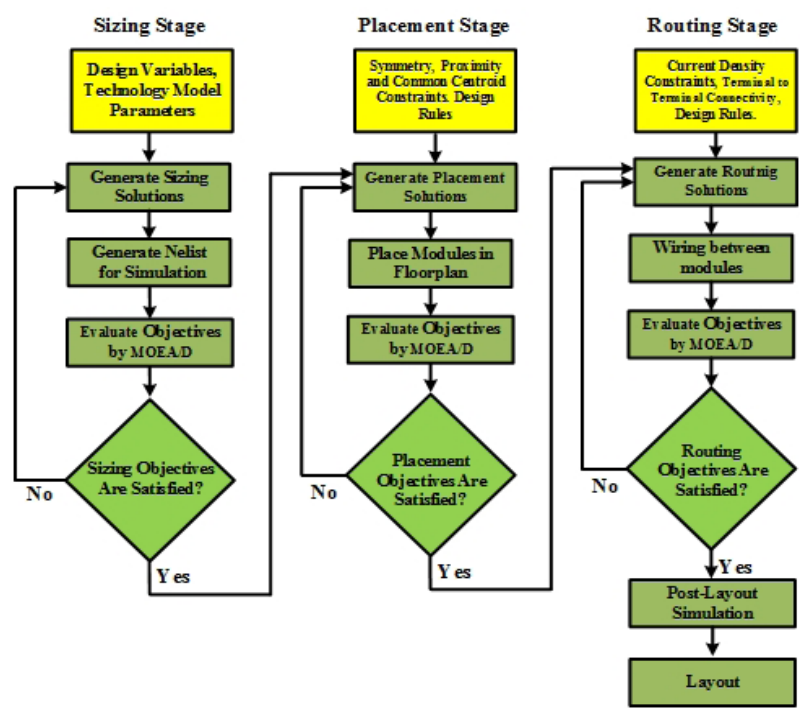

Figure 3: Flowchart of the proposed analog layout generation method.

\subsection{Proposesd layout generation method}

The flowchart of the proposed analog layout generation method is shown in Figure3. The proposed method details are as follows:

\section{Sizing Stage}

Inputs: Design variables such as width, length, and the number of fingers of the MOS transistors, bias voltages, the values of the passive components, and technology models parameters.

\section{Sizing Procedure:}

Step 1: Solutions are provided using the optimization algorithm. It this paper, it is performed by the MOEA/D. Step 2: After providing the solutions by the MOEA/D, a netlist is created. Schematic simulation should be performed to evaluate the circuit specifications. In this paper, simulation is performed using HSPICE software. Step 3: Specifications of the circuit such as DC-gain, phase margin $(P M)$, power dissipation $\left(P_{\text {diss }}\right)$, settling time $(\mathrm{ST})$, slew rate (SR) and unity-gain-bandwidth (UGBW), are evaluated from the schematic simulation results.

Step 4: If the specifications are satisfied, the solutions are given to the placement stage. Otherwise, new solutions should be found by the MOEA/D.

\section{Placement Stage}

Inputs: Symmetry, proximity and common centroid constraints, design rules, device sizes from the sizing stage. Placement Procedure:

Step 5: The coordinates of the left-bottom corners of the modules are chosen by the MOEA/D.

Step 6: The modules are placed in the floorplan using the solutions obtained from the previous step. 
Step 7: In this step, the placement objectives are evaluated. A compact floorplan should be produced in the placement stage by simultaneous consideration of design rules and the other constraints.

Step 8: If the placement objectives are satisfied, the solutions are given to the routing stage. Otherwise, new solutions should be provided by the EMO.

\section{Routing Stage}

Inputs: Current-density constraints, terminal to terminal connectivity, design rules, floorplan from the placement stage.

\section{Routing Procedure:}

Step 9: In this step, the obtained floorplan from the placement stage is given to the router. The solutions are generated by the router.

Step 10: Based on the segment positions, wiring between terminals are performed.

Step 11: The routing objectives according to the Section 3.2 are evaluated.

Step 12: If the routing objectives are satisfied, the solutions are given to the next stage. Otherwise, MOEA/D is looking for new solutions.

Step 13: After extracting the parasitics of the obtained layout, post-layout simulation is performed. Parasitics extraction are done using a resistance-capacitance (RC) $\pi$ model that is suggested in Ref [38]. If post-simulation on routing stage somehow fails, to find new solutions the routing stage should be repeated. It usually takes a few repetitions to get the answer.

Step 14: The final layout of the proposed method is drawn.

\section{Performance Evaluation}

The proposed analog layout generation method is performed in a $0.18 \mu \mathrm{m} 1.8 \mathrm{~V}$ CMOS technology. An automatic MATLAB toolbox is provided which is connected to HSPICE. MOEA/D is implemented in MATLAB R2016b version and is tested on Intel corei5-4460 CPU @ 3.2 $\mathrm{GHz}$ with 16 GB RAM. Operational amplifiers (op-amps) can be considered as an essential block in many mixedmode systems [39-42].The proposed method is applied to three-stage amplifier and two-stage class-AB operational trans-conductance amplifier (OTA).

\subsection{Three-stage amplifier}

In Ref [42], optimization of the settling performance of a three-stage amplifier shown in Figure 4 is studied. In the following, designing the three-stage op-amp shown in Figure 4 is presented.

$$
f(x)=\left(f_{1}(x), f_{2}(x), f_{3}(x), f_{4}(x), f_{5}(x), f_{6}(x)\right)
$$

In the above equation , $f_{1}(x)=-1 x D C-$ gain, $f_{2}(x)=-1 x P M$, $f_{3}(x)=-1 x \cup G B W, f_{4}(x)=-1 x S R, f_{5}(x)=-P_{\text {diss' }}$ and $f_{6}(x)=S T . x$ represents the design variables. Designing is performed using minimum channel length transistor $(0.18 \mu \mathrm{m})$, and the widths of the MOS transistors are chosen as $2 \mu \mathrm{m} \leq \mathrm{Wj}$ $\leq 150 \mu \mathrm{m}$. MOEA/D is executed with a population of 100 individuals with 250 iterations. It should be noted that in each iteration, the $z^{*}$ in selected as the minimum of the $z^{*}$ and the cost function. One solution to the sizing result is shown in Table 1. The placement and routing results are done by the layout generator and the result is depicted in Figure 5 . The area of the layout is $34 \mu \mathrm{m} \times 32 \mu \mathrm{m}$. The total number of wires is 23 . In this Figure, metal 1 and metal 2 are shown by green and yellow colors, respectively.

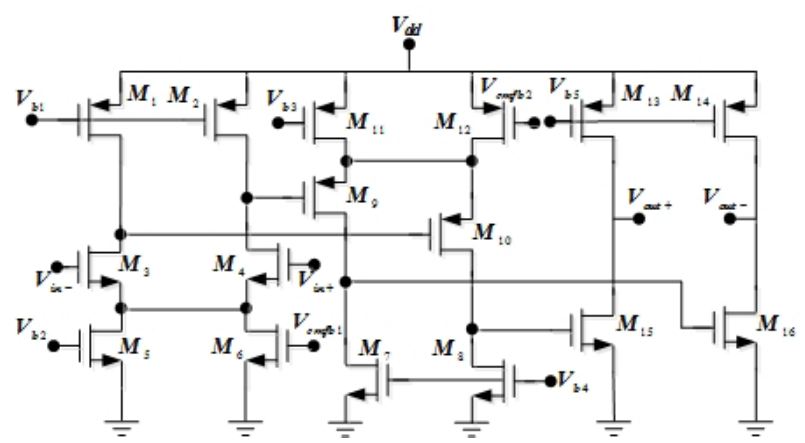

Figure 4: CMOS Three-Stage op-amp [42].

Table 1: Size of transistors for the three-stage op-amps.

\begin{tabular}{|l|l|}
\hline Parameter & Value \\
\hline$(\mathrm{W} / \mathrm{L})_{1,2}$ & $10 \times 10 \mu \mathrm{m} / 0.18 \mu \mathrm{m}$ \\
\hline$(\mathrm{W} / \mathrm{L})_{3,4}$ & $8 \times 6.25 \mu \mathrm{m} / 0.18 \mu \mathrm{m}$ \\
\hline$(\mathrm{W} / \mathrm{L})_{5,6}$ & $10 \times 5 \mu \mathrm{m} / 0.18 \mu \mathrm{m}$ \\
\hline$(\mathrm{W} / \mathrm{L})_{7,8}$ & $16 \times 3.125 \mu \mathrm{m} / 0.18 \mu \mathrm{m}$ \\
\hline$(\mathrm{W} / \mathrm{L})_{9,10}$ & $16 \times 6.25 \mu \mathrm{m} / 0.18 \mu \mathrm{m}$ \\
\hline$(\mathrm{W} / \mathrm{L})_{11,12}$ & $16 \times 12.5 \mu \mathrm{m} / 0.18 \mu \mathrm{m}$ \\
\hline$(\mathrm{W} / \mathrm{L})_{13,14}$ & $10 \times 20 \mu \mathrm{m} / 0.18 \mu \mathrm{m}$ \\
\hline$(\mathrm{W} / \mathrm{L})_{15,16}$ & $10 \times 4 \mu \mathrm{m} / 0.18 \mu \mathrm{m}$ \\
\hline
\end{tabular}

The open-loop frequency responses of the designed op-amp in demonstrated in Figure 6. The results show that the DC-gain is $72 \mathrm{~dB}$ after post-layout simulation. UGBW and PM of the Op-Amp are $720 \mathrm{MHz}$ and $82^{\circ}$, respectively. A $0.4 \mathrm{~V}$ step voltage is applied to the op-amp and the $1 \%$ ST is calculated. The obtained step response is shown in Figure 7. The results shown in the Figures 6 and 7 are also obtained by the Cadence software. It should be mentioned that the proposed method results are consistent with the Cadence software. 


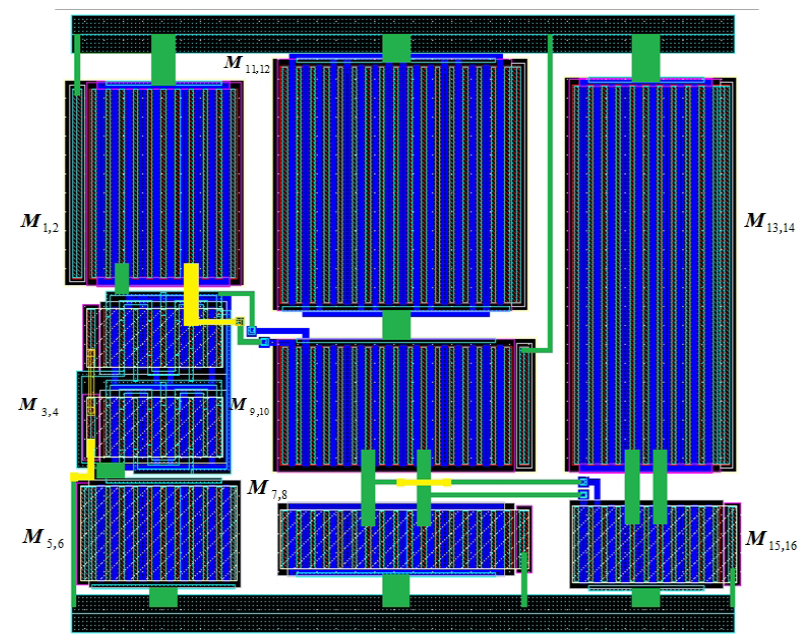

Figure 5: The three-stage amplifier layout generated by the proposed method.

(a)

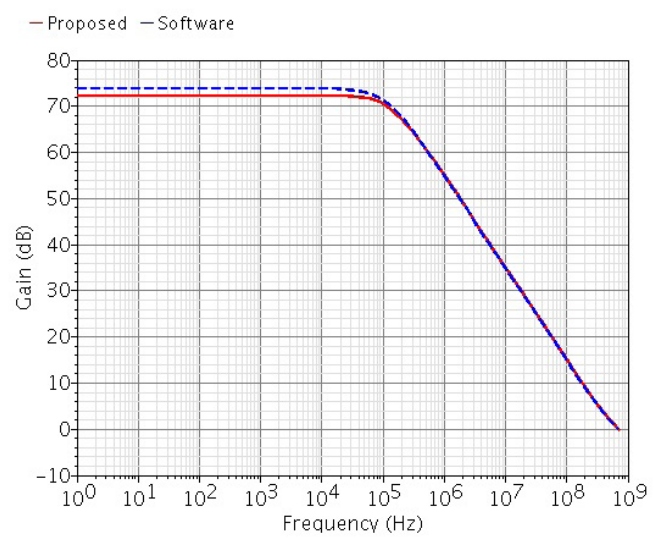

(b)

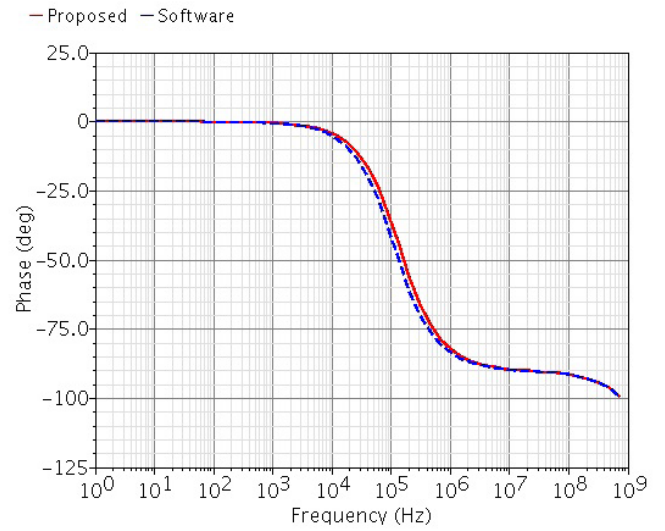

Figure 6: Three-stage op-amp frequency response: (a) magnitude, (b) phase.

The Pareto optimal fronts (POFs) of the three-stage opamp including ST versus $P_{\text {diss }}$ DC-Gain versus $P_{\text {diss }}$, $S R$ versus $P_{\text {diss }}$ and UGBW versus PM are shown in Figure 8 . As can be seen from the Figure 8.a, the proposed method can achieve better ST compared to the existing methods [14], [31]. Table 2 reports the comparisons of post-layout simulation results of the proposed method with the ex-

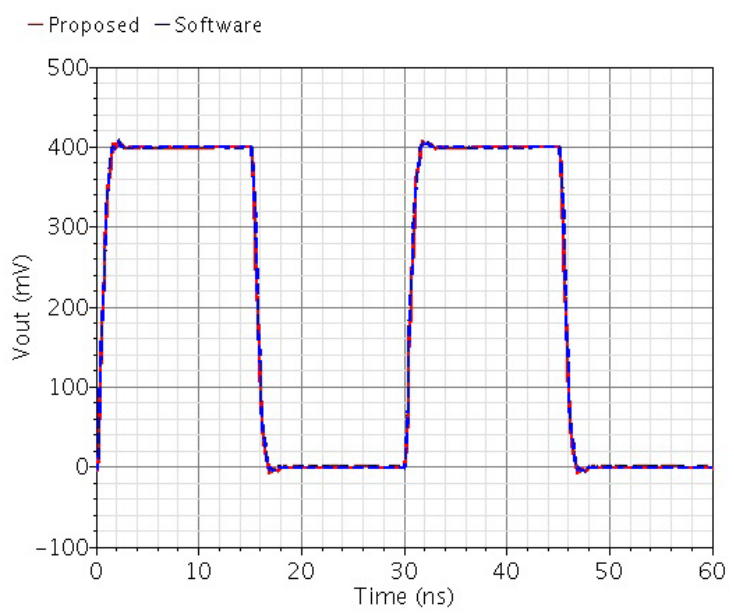

Figure 7: Three-stage op-amp step response.

isting methods. The main advantage of the proposed method compared with the existing methods is that the sizing stage is not considered in the existing methods. In addition, a set of solutions are provided by MOEA/D in the proposed method compared to the single solution in the [31]. In the [31], area utilization and number of bends are not included for the placement and routing stages, respectively. It is essential to define initial wires and some operators for the routing stage in the [14]. The ST, layout area and also area utilization of the proposed method is better than the existing methods with the cost of a little increase in the runtime. Therefore, the proposed method is more suitable for automatic analog layout generation.

Table 2: Comparisons of the post-layout simulation results for three-stage amplifier.

\begin{tabular}{|l|l|c|c|c|}
\multirow{2}{*}{ No. } & \multirow{2}{*}{ Specifications } & \multicolumn{3}{|c|}{ Post-layout performance } \\
\cline { 3 - 5 } & & $\begin{array}{c}\text { This } \\
\text { work }\end{array}$ & {$[31]$} & {$[14]$} \\
\hline 1 & $1 \%$ Settling time $(\mathrm{ns})$ & $<4.4$ & 4.9 & 5.2 \\
\hline 2 & Total runtime $(\mathrm{s})$ & 1531 & 1266 & 1134 \\
\hline 3 & Layout Area $(\mathrm{mm} 2)$ & 0.0011 & 0.0013 & 0.0014 \\
\hline 4 & Area Utilization $(\%)$ & 88 & 82 & 76 \\
\hline
\end{tabular}

\subsection{Two-stage class-AB OTA}

In Ref [41], a two-stage class-AB OTA is suggested. By increasing the trans-conductance of the first stage, DCgain is enhanced. Non-linear current mirror boosts the current of the second stage. As a result, the SR is improved. The OTA is shown in Figure 9. Analytical equations are provided in the Ref. 41 . The placement and routing results are obtained from the proposed method and the result is shown in Figure 10. The area of the layout is $130 \mu \mathrm{m} \times 195 \mu \mathrm{m}$. The POFs of the two-stage class- 
(a)

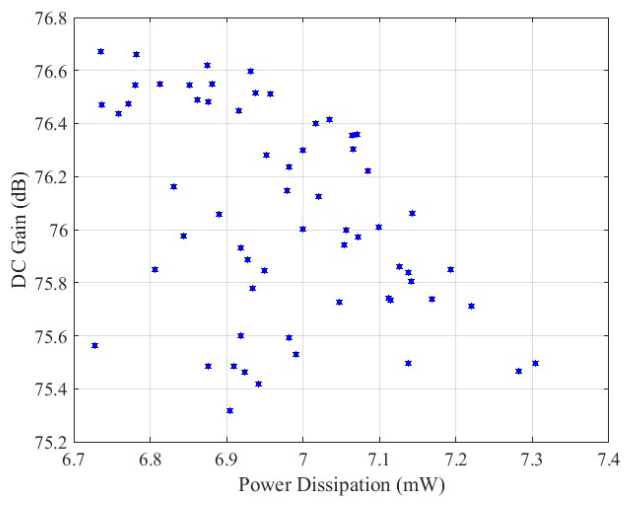

(b)

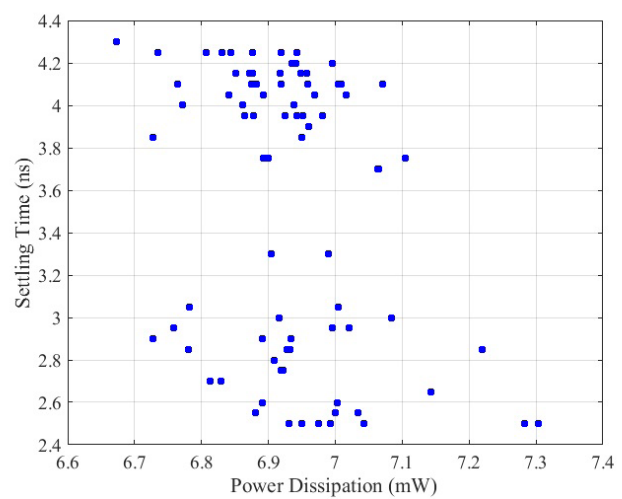

(c)

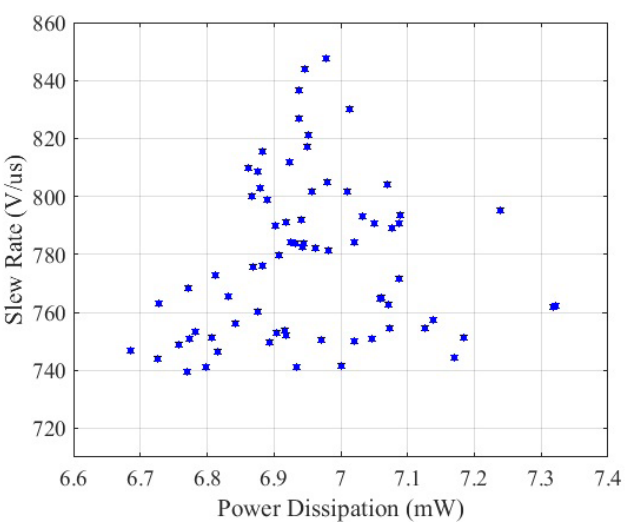

(d)

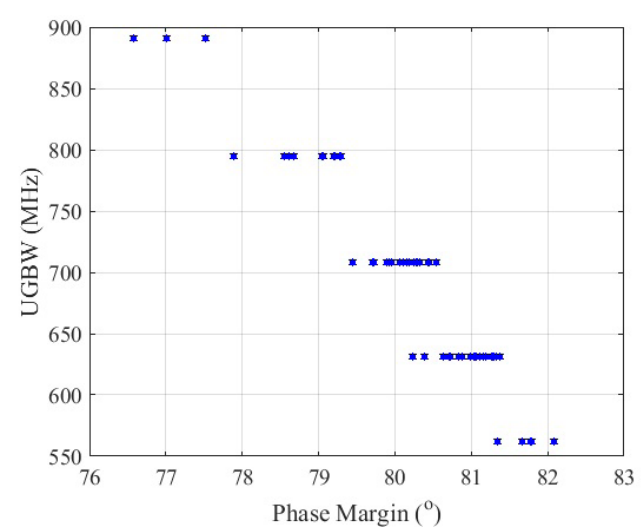

Figure 8: POFs of the three-stage: (a) ST versus $P_{\text {diss' }}$ (b) DC-gain versus $P_{\text {diss' }}$ (c) SR versus $P_{\text {diss' }}$ (d) UGBW versus PM.
$A B$ OTA including DC-Gain versus $P_{\text {diss' }}$ SR versus $P_{\text {diss }}$ and UGBW versus PM are shown in Figure 11. The results show that DC-Gain and UGBW reach $84 \mathrm{~dB}$ and $68 \mathrm{MHz}$, respectively. In addition, according to the $\mathrm{PM}$ values, the system is stable. Table 3 indicates the comparisons of post-layout simulation results of the proposed method with the existing methods for the two-stage class-AB OTA. Simulation results indicate that the proposed method outperforms the existing methods in terms of DC-gain, SR, layout area and area utilization.

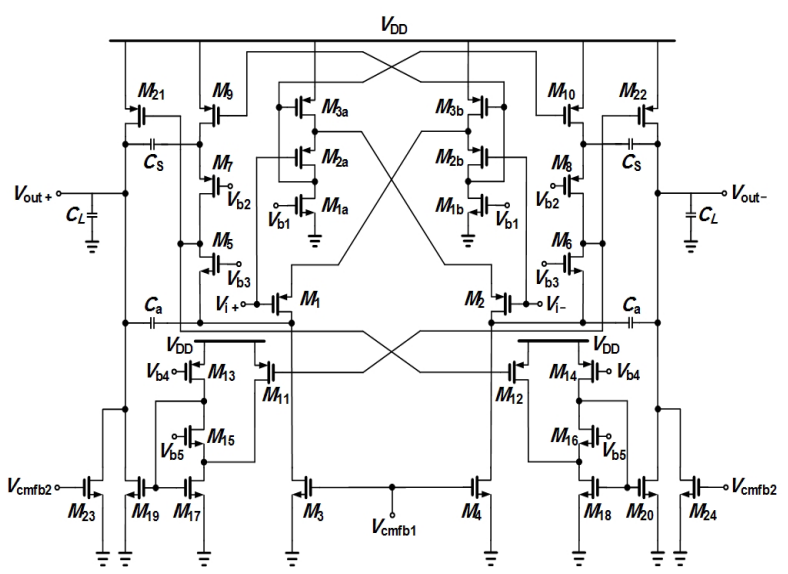

Figure 9: CMOS two-stage class-AB OTA [41].

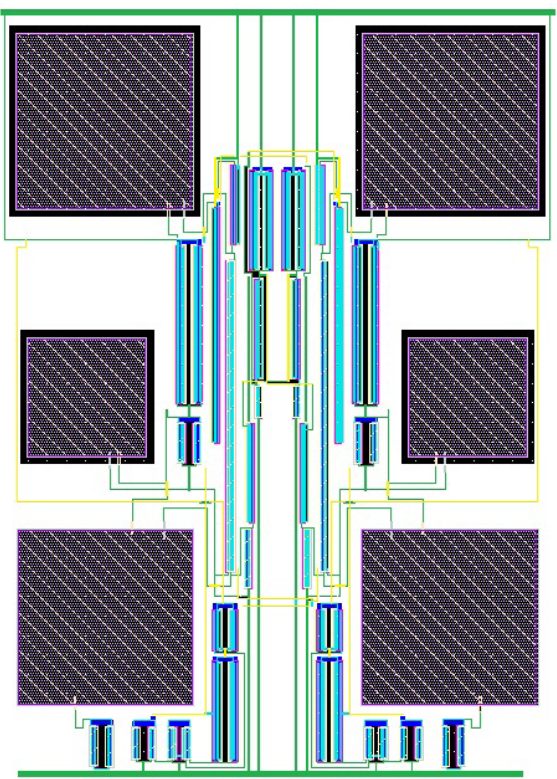

Figure 10: The two-stage class-AB OTA layout generated by the proposed method. 
(a)

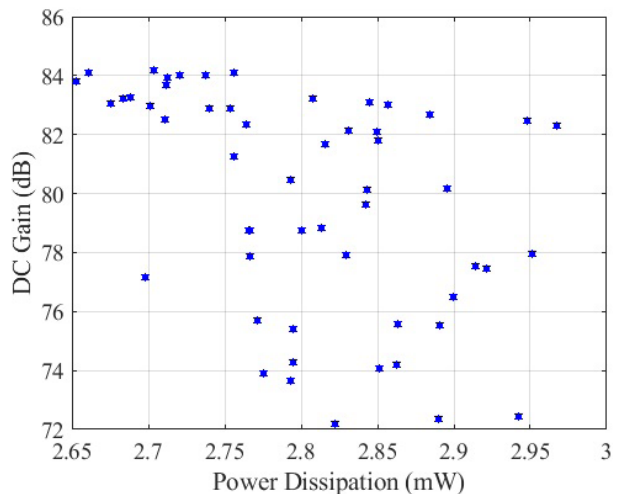

(b)

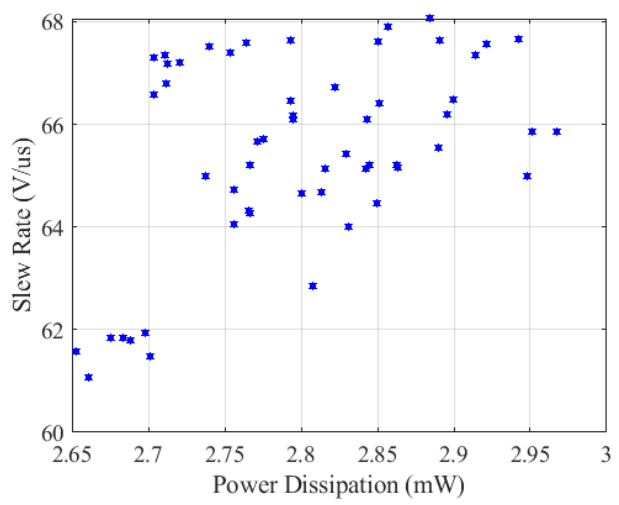

(c)

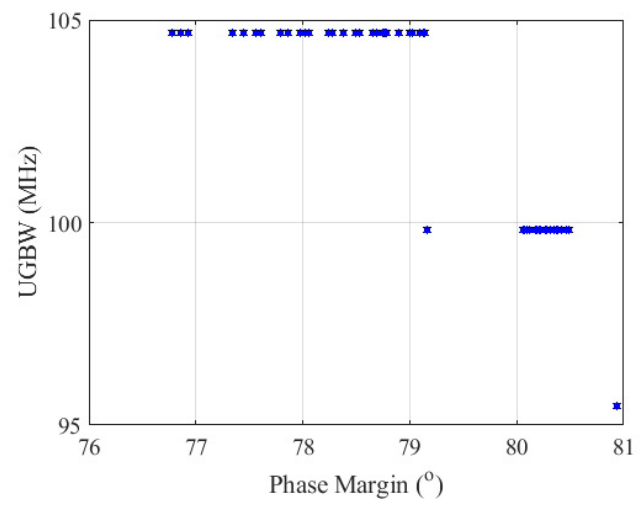

Figure 11: POFs of the two-stage class-AB OTA: (a) DCgain versus $P_{\text {diss' }}$ (b) $S R$ versus $P_{\text {diss' }}$ (c) UGBW versus PM.

Table 3: Comparisons of the post-layout simulation results for the two-stage class-AB OTA.

\begin{tabular}{|c|c|c|c|c|}
\hline \multirow{2}{*}{ No. } & \multirow{2}{*}{ Specifications } & \multicolumn{3}{|c|}{ Post-layout performance } \\
\hline & & This work & [31] & [14] \\
\hline 1 & DC-gain (dB) & 84 & 80 & 76 \\
\hline 2 & Slew Rate $V / \mu \mathrm{s}$ & 68 & 65 & 61 \\
\hline 3 & Total runtime (s) & 2143 & 1785 & 1610 \\
\hline 4 & Layout Area $(\mathrm{mm} 2)$ & 0.025 & 0.027 & 0.028 \\
\hline 5 & Area Utilization (\%) & 89 & 84 & 78 \\
\hline
\end{tabular}

\section{Conclusions}

A new automatic placement and routing method for layout generation of op-amps has been presented in this paper. In addition, the sizing has been performed automatically. Layout effects including parasitics and geometry effects have been considered. A compact floorplan has been generated in the placement stage by considering a set of constraints. A router has been suggested to generate wires automatically. MOEA/D has been utilized for optimization which is suitable for multi-objective optimization problems. In order to evaluate the performance of the proposed method, some simulations have been carried out and the results indicated the efficiency of the automatic analog layout generation method.

\section{Conflicts of Interest}

The authors declare no conflict of interest.

\section{Acknowledgement}

This work wae supported by Shahid Rajaee Teacher Training University.

\section{References}

1. T. McConaghy, P. Palmers, M. Steyaert, G. G. E. Gielen, Trustworthy genetic programming-based synthesis of analog circuit topologies using hierarchical domain-specific building blocks, IEEE Trans. Evolutionary Computation 15 (2011) 557-570.

2. Ž. Rojec, A. Bürmen, I. Fajfar, Analog circuit topology synthesis by means of evolutionary computation, Engineering Applications of Artificial Intelligence, Volume 80, 2019, Pages 48-65, ISSN 0952-1976,

https://doi.org/10.1016/j.engappai.2019.01.012

3. Samin Ebrahim Sorkhabi, Lihong Zhang, Automated topology synthesis of analog and RF integrated circuits: A survey. Integration, Volume 56, 2017.

4. Slezak, J, Petrzela, Evolutionary Synthesis of Cube Root Computational Circuit Using Graph Hybrid Estimation of Distribution Algorithm. RADIOENGINEERING, 1210-2512 APR, 2014.

5. Y. Gua, et al., Rapid and accurate method for resizing CMOS operational amplifiers, Analog Integrated Circuits and Signal Processing, 99 (2019), 447-454.

https://doi.org/10.1007/s10470-019-01428-8 
6. R. Martins, et al., Many-Objective Sizing Optimization of a Class-C/D VCO for Ultralow-Power loT and Ultra low-Phase-Noise Cellular Applications, IEEE Transactions on Very Large Scale Integration (VLSI) Systems, 27 (2019), 69-82.

http://doi.org/10.1109/TVLSI.2018.2872410

7. S. Park, S. Raman, Analysis and Optimization of Multisection Capacitive DACs for Mixed-Signal Processing, IEEE Transactions on Very Large Scale Integration (VLSI) Systems, 27 (2019), 679 - 690. https://doi.org/10.1109/TVLSI.2018.2888593

8. D. Martev, S. Hampel, U. Schlichtmann, Automated Phase-Noise-Aware Design of RF Clock Distribution Circuits, IEEE Transactions on Very Large Scale Integration (VLSI) Systems, 26 (2018), 23952405.

https://doi.org/10.1109/TVLSI.2018.2864316

9. W. Lyu, et al., An Efficient Bayesian Optimization Approach for Automated Optimization of Analog Circuits, IEEE Transactions on Circuits and Systems I: Regular Papers, 65 (2018), 1954-1967.

10. S. M. Anisheh, C. Dadkhah, A two-stage method for optimizing the parameters of CMOS operational amplifiers based on evolutionary algorithm, International Journal on Computer Science and Engineering, 14 (2017), 1-10.

11. N. Khalil, et al., An intelligent technique for generating equivalent gyrator circuits using Genetic Algorithm, Microelectronics Journal, 46 (2015), 1060-1068.

https://doi.org/10.1016/j.mejo.2015.09.004

12. M. H. Maghami, F. Inanlou, R. Lotfi, SimulationEquation-Based Methodology for Design of CMOS Amplifiers Using Geometric Programming, Proc. of $15^{\text {th }}$ IEEE International Conference on Electronics, Circuits and Systems (2008), 360-363. https://doi.org/10.1109/ICECS.2008.4674865

13. H-J. Chang, et al., Layout-aware Analog Synthesis Environment with Yield Consideration, Proc. of $16^{\text {th }}$ International Symposium on Quality Electronic Design (2015), 589-593.

14. R. Martins, N. Lourenco, N. Horta, LAYGEN II-Automatic Layout Generation of Analog Integrated Circuits, IEEE Transactions on Computer-Aided Design of Integrated Circuits and System, 32 (2013), 1641-1654.

https://doi.org/10.1109/TCAD.2013.2269050

15. E. Yilmaz, G., Dündar, Analog Layout Generator for CMOS Circuits, IEEE Transactions on ComputerAided Design, 28 (2009), 32-45. https://doi.org/10.1109/TCAD.2008.2009137

16. A. Agarwal, R. Vemuri, Layout-Aware RF Circuit Synthesis Driven by Worst Case Parasitic Corners, Proc. of International Conference on Computer Design (2005), 1-6.

https:/doi.ieeecomputersociety.org/10.1109//CCD.2005.68
17. A. Ferreira, et al., Automated Analog IC Design Constraints Generation for a Layout-Aware Sizing Approach, Proc. of $13^{\text {th }}$ International Conference on Synthesis, Modeling, Analysis and Simulation Methods and Applications to Circuit Design (2016), 1-4.

https://doi.org/10.1109/SMACD.2016.7520740

18. N. Lourenco, et al., AIDA: Layout-aware analog circuit-level sizing with in-loop layout generation, Integration, the VLSI Journal, 55 (2016), 316-329. https://doi.org/10.1016/j.vlsi.2016.04.009

19. A. Toro-Frías, et al., Layout-aware pareto fronts of electronic circuits, Proc. of European Conference on Circuit Theory and Design (2011), 345-348. https://doi.org/10.1109/ECCTD.2011.6043357

20. T. Liao, L. Zhang, Parasitic-aware GP-based manyobjective sizing methodology for analog and RF integrated circuits, Proc. of Asia and South Pacific Design Automation Conference (2017), 475-180.

21. P. Vancorenland, et al., A layout-aware synthesis methodology for RF circuits, Proc. of IEEE/ACM ICCAD (2001), 358-362.

22. S. Youssef, et al., A Python-Based Layout-Aware Analog Design Methodology for Nanometric Technologies, Proc. of IEEE 6th Int. IDT (2011), 62-67.

23. Y-C. Liao, et al., LASER: layout-aware analog synthesis environment on laker, Proc. of $23^{\text {rd }}$ ACM GLSVLSI (2013), 107-112. https://doi.org/10.1145/2483028.2483071

24. R. Castro-Lopez, et al., An Integrated Layout-Synthesis Approach for Analog ICs, IEEE Transactions on Computer Aided Design, 27 (2008), 1179-1189. https://doi.org/10.1109/TCAD.2008.923417

25. G. Berkol, et al. A Two-Step Layout-in-the-loop Design Automation Tool, Proc. of IEEE $13^{\text {th }}$ NEWCAS (2015), 1-4.

26. A. Agarwal, et al., Fast and accurate parasitic capacitance models for layout-aware synthesis of analog circuits, Proc. of $41^{\text {st }}$ Conference on Design Automation (2004), 145-150.

https://doi.org/10.1145/996566.996610

27. K. Choi, D. Allstot, S. Kiaei, Parasitic-aware synthesis of RF CMOS switching power amplifiers, Proc. of IEEE International Symposium on Circuits and Systems (2002), 269-272.

28. G. Zhang. et al., A synthesis flow toward fast parasitic closure for radio-frequency integrated circuits, Proc. of Design Automation Conference (2004), 155-158. https://doi.org/10.1145/996566.996612

29. M. Ranjan, et al., Fast, layout-inclusive analog circuit synthesis using precompiled parasitic-aware symbolic performance models, Proc. of Des., Autom. Test Eur. Conf. (2004), 604-609.

30. A. Ahmed, L. Zhang, Fast parasitic-aware synthesis methodology for high-performance analog 
circuits, Proc. of IEEE International Symposium on Circuits and Systems (2012), 2155-2158. https://doi.org/10.1109/ISCAS.2012.6271714

31. S. M. Anisheh, H. Shamsi, Placement and Routing Method for Analogue Layout Generation Using Modified Cuckoo Optimization Algorithm, IET Circuits Devices \& Systems, 12 (2018), 532-541. https://doi.org/10.1049/iet-cds.2017.0111

32. Mirhoseini, A., Goldie, A., Yazgan, M. et al. A graph placement methodology for fast chip design. Nature 594, 207-212 (2021).

https://doi.org/10.1038/s41586-021-03544-w

33. Y. Yan Tan, et al., MOEA/D+uniform design: A new version of MOEA/D for optimization problems with many objectives, Computers \& Operations Research, 40 (2013), 1648-1660. https://doi.org/10.1016/j.cor.2012.01.001

34. I. Giagkiozis, et al., Generalized Decomposition and Cross Entropy Methods for Many-Objective Optimization, Information Science, 282 (2014), 363-387.

35. Q. Zhang, H. Li, MOEA/D: A Multiobjective Evolutionary Algorithm Based on Decomposition, IEEE Transactions on Evolutionary Computation, 11 (2007), 712-731. https://doi.org/10.1109/TEVC.2007.892759

36. Y. Jin, T. Okabe, and B. Sendho, Adapting Weighted Aggregation for Multiobjective Evolution Strategies, International Conference on Evolutionary Multi-Criterion Optimization (2001), 96-110.

37. H. E. Graeb, Analog Layout Synthesis, New York, NY, USA: Springer, 2011.

38. L. Zhang, Z. Liu, Directly performance-constrained template-based layout retargeting and optimization for analog integrated circuits, Integration, the VLSI journal, 44 (2011), 1-11. https://doi.org/10.1016/j.vlsi.2010.09.003

39. S. M. Anisheh, H. Shamsi, Two-stage class-AB OTA with enhanced DC gain and slew rate, International Journal of Electronics Letters, 5 (2017), 438-448. https://doi.org/10.1080/21681724.2016.1253780
40. S. M. Anisheh, H. Shamsi, M. Mirhassani, Positive Feedback Technique and Split-Length Transistors for DC-Gain Enhancement of Two Stage OpAmps, IET Circuits Devices \& Systems, 11 (2017), 605-612.

https://doi.org/10.1049/iet-cds.2016.0416

41. S. M. Anisheh, H. Abbasizadeh,, H. Shamsi, C. Dadkhah, K. Y. Lee, 84 dB DC-gain two-stage class-AB OTA, IET Circuits Devices \& Systems, 13 (2019), 614621.

https://doi.org/10.1049/iet-cds.2018.5038

42. R. Nguyen, B. Murmann, The design of fast-settling three-stage amplifiers using the open-loop damping factor as a design parameter, IEEE Transactions on Circuits and Systems-I: Regular Papers, 57 (2010), 1244-1254. https://doi.org/10.1109/TCSI.2009.2031763

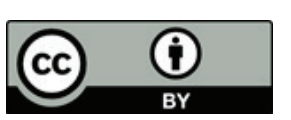

Copyright (c) 2021 by the Authors. This is an open access article distributed under the Creative Commons Attribution (CC BY) License (https://creativecommons.org/licenses/by/4.0/), which permits unrestricted use, distribution, and reproduction in any medium, provided the original work is properly cited.

Arrived: 27. 04. 2021

Accepted: 02. 09. 2021 